Research Paper

\title{
Hypoxia-Induced Deregulation of miR- 126 and Its Regulative Effect on VEGF and MMP-9 Expression
}

\author{
Panpan $\mathrm{Ye}^{1,2}$, Jian Liu ${ }^{1,2}$, Fengying $\mathrm{He}^{1,2}$, Wen $\mathrm{Xu}^{1,2}$, Ke $\mathrm{Yao}^{1,2} \bowtie$ \\ 1. Eye Center, Second Affiliated Hospital, School of Medicine, Zhejiang University; \\ 2. Zhejiang Provincial Key Lab of Ophthalmology, China.
}

$\triangle$ Corresponding author: Ke Yao, Eye Center, Second Affiliated Hospital, College of Medicine, Zhejiang University, Hangzhou, China; Zhejiang Provincial Key Lab of Ophthalmology, China. E-mail: xlren@zju.edu.cn.

() Ivyspring International Publisher. This is an open-access article distributed under the terms of the Creative Commons License (http://creativecommons.org/ licenses/by-nc-nd/3.0/). Reproduction is permitted for personal, noncommercial use, provided that the article is in whole, unmodified, and properly cited.

Received: 2013.08.03; Accepted: 2013.11.25; Published: 2013.12.10

\begin{abstract}
Objective: miR-126, the miRNA considered to be specially expressed in endothelial cells and hematopoietic progenitor cells, is strongly associated with angiogenesis. The purpose is to evaluate the role of miR-126 in hypoxia-induced angiogenesis and the possible mechanisms. Methods: The expression of miR-126 was detected in hypoxia-treated RF/6A cells and diabetic retinas using real-time PCR. The miR-126 was up- or down-regulated by transfecting miR-126-mimics or inhibitors into RF/6A cells. Cell cycle analysis was performed using flow cytometry. The protein levels of vascular endothelial growth factor (VEGF) and matrix metalloproteinase-9 (MMP-9) were assessed by immunoblotting. Results: A significantly decreased expression of miR-126 was found in hypoxia-treated RF/6A cells in a time-dependent manner compared with normoxic condition. The expression of miR-126 was also reduced in the retina tissue of streptozotocin-induced diabetic rats. The expression of VEGF and MMP-9 proteins was increased in hypoxia-induced RF/6A cells. In the functional analysis, miR-126-mimic significantly reduced the percentage of RF/6A cells in $\mathrm{S}$ phases compared with the negative control under hypoxic conditions. Furthermore, the VEGF and MMP-9 protein levels were sharply decreased in hypoxia-induced RF/6A cells pretreated with miR-126-mimics and increased in the cells pretreated with miR-126-inhibitors. Conclusions: miR-126 is down-regulated under hypoxic condition both in vitro and in vivo and may halt the hypoxia-induce neovascularization by suspending the cell cycle progression and inhibiting the expression of VEGF and MMP-9.
\end{abstract}

Key words: MicroRNA-126, Hypoxia, Vascular endothelial growth factor, Matrix metalloproteinase-9, Diabetic retinopathy.

\section{Introduction}

Retinal neovascularization is one of the leading causes of visual impairment in many diseases such as proliferative diabetic retinopathy (PDR), central retinal vein occlusion (CRVO) and retinopathy of prematurity (ROP) [1]. Although laser photocoagulation can serve as an efficient short-term therapy, the side effects (e.g. loss of peripheral vision, reduction of night vision) greatly limit its value and, more importantly, there is still a lack of methods for a final cure.

As is well known, retinal tissue is the most met- abolically active human tissue and highly sensitive to hypoxia [2]. Hypoxia is an important pathophysiological signal and can cascade down in a series of angiogeneic processes in retinal neovascularization diseases. It has been reported that the most important feature of endothelial cell hypoxia is its triggering of angiogenesis, which involves basal membrane degradation, endothelial cell proliferation, migration, and neovascularization [3]. Therefore, exploring the biological alterations of endothelial cells under a hypoxia condition can be helpful to better understand the 
mechanism of retinal neovascularization diseases and provide potential molecular therapies.

MicroRNAs (miRNAs) are a family of small non-coding single-strand RNAs and act as gene regulators through translational repression or messenger RNA (mRNA) degradation via binding to target sites in the 3'-untranslated regions (UTR) of protein-coding transcripts. They regulate a variety of biological processes including cell differentiation, proliferation, apoptosis, metabolism and neoplastic transformation [4]. So far, more than 1400 human miRNA sequences (1424 miRbase 17.0) have been identified [5], and miRNA functional characterization has become a major interest in biology and medicine with implications for the detection and treatment of different pathologies.

In the vascular system, miRNAs are abundant and play key roles in vascular development and vascular system diseases such as tumor angiogenesis and ocular neovascularization [6, 7]. miR-126, the only miRNA considered to be specially expressed in endothelial cells and hematopoietic progenitor cells, is strongly associated with angiogenesis [8,9]. It has been found that deletion of miR-126 causes a loss of vascular integrity and defects in endothelial progenitor cells-mediated functional vascular properties [9-11]. In this study, we aim to evaluate the role of miR-126 in hypoxia-induced angiogenesis and the possible mechanisms.

\section{Materials and Methods}

\section{Cell culture}

Monkey chorioretinal vessel endothelial cells (RF/6A) were obtained from Shanghai Institute of Biochemistry and Cell Biology of the Chinese Academy of Sciences (Shanghai, China). All cells were grown in Eagle's minimum essential medium (EMEM) (Jinuo, Hangzhou, China), supplemented with 10\% fetal bovine serum (FBS) (Invitrogen-Gibco, Carlsbad, CA). The cells were maintained at $37^{\circ} \mathrm{C}$ in a humidified $5 \% \mathrm{CO}_{2}$ atmosphere.

\section{Cell culture under hypoxic conditions}

$\mathrm{RF} / 6 \mathrm{~A}$ cells were placed in serum-free medium. One milliliter of cells $\left(1 \times 10^{5}\right.$ cells/well $)$ were plated into one well of a six-well culture plate. Hypoxic cultures were transferred for various time periods ( $1 \%$ $\mathrm{O}_{2}, 5 \% \mathrm{CO}_{2}, 94 \% \mathrm{~N}_{2}$ labeled as hypoxia) in a hypoxic incubator (BioSpherix, Redfield, NY). The cells were harvested in hypoxic conditions to avoid reoxygenation artifacts. Parallel cultures were kept in normal oxygen levels (labeled as normoxia).

\section{Animals and treatment with streptozotocin}

Male Wistar rats weighing 130 to $150 \mathrm{~g}$ were housed in suspended wire-bottom cages in a room kept at $25 \pm 2{ }^{\circ} \mathrm{C}$ with a $12: 12 \mathrm{~h}$ light-dark cycle and were provided with food and water. The animals were randomly divided into two groups. For the induction of diabetes, streptozotocin (STZ) (Sigma Chemical Co., St. Louis, MO) was dissolved in $0.01 \mathrm{M}$ citrate buffer, $\mathrm{pH} 4.5$, and was injected within 5 min of its preparation. The rats fasted overnight and then were anesthetized with isoflurane and were injected with STZ in the jugular vein at a dose of $45 \mathrm{mg} / \mathrm{kg}$ bodyweight. The control rats were injected with the citrate buffer. Blood glucose levels were measured at $24 \mathrm{~h}$ after the injection of STZ or citrate buffer. Only animals with blood glucose values $\geq 400 \mathrm{mg} / \mathrm{dl}$ were used. The STZ-injected and control rats were sacrificed by rapid cervical dislocation at the indicated time points after treatment. The retinas were dissected and used for the analysis of miRNA. The care and treatment of the animals received prior institutional approval from the Ethical Commission of Ethics in Animal Research of School of Medicine at Zhejiang University.

\section{Immunohistochemistry}

Retina was fixed in $10 \%$ phosphate buffer formalin and cut into thin sections $(\mathrm{n}=7)$. Retinal slides were stained using hemotoxylin and eosin (HE) stain and observed under $\times 200$ magnification.

\section{Cell treatment with miRNA inhibitor or mimics}

$\mathrm{RF} / 6 \mathrm{~A}$ cells were treated with miR-126 mimic (miR-126-mimic) or miR-126 inhibitor (miR-126-inhibitor) (Invitrogen, CA, USA) using Lipofectamine 2000 in OptiMEM I Reduced Serum Medium (Invitrogen). Total RNA and cell lysate were collected for the indicated assays. miRNA mimics negative control (mimic-NC) and miRNA inhibitor negative control (inhibitor-NC) (Invitrogen) were severed as negative controls in the experiments respectively. RF/6A cells were pretreated with miR-126-mimics, miR-126-inhibitors, or their negative controls for $6 \mathrm{~h}$ of normoxia and then transferred to hypoxia for indicated experiments.

\section{RNA extraction, cloning of miRNAs and re- al-time quantitative polymerase chain reaction (PCR)}

Total RNA was extracted from cells or tissues using Trizol (Invitrogen) according to the manufacturer's instructions. Total RNA was reverse-transcribed using Superscript III reverse transcriptase and oligo(dT) primer (Invitrogen). Real time PCR was performed and analyzed in ABI PRISM 7500 Sequence Detection System (Applied Biosystem, 
Carlsbad, California, USA) with SYBR green ready mix (Applied Biosystem) and SDS 2.1 software (Applied Biosystems). All reactions were measured in triplicates in a final volume of $10 \mu \mathrm{L}$. Cycling conditions were chosen according to the manufacturer's protocols. For miRNA detection, the relative level of miRNA was calculated against U6 RNA (internal control) using the $2^{-\Delta \Delta C t}$ method.

\section{Cell cycle analysis}

Cells were reseeded into 6-well plates and supplemented with miR-126-mimic, miR-126-inhibitor or their negative controls. Detached cells were collected by centrifugation, resuspended in phosphate-buffered saline (PBS) supplemented with DNA Prep LPR (Coulter DNA-Prep reagents kit, Beckman Coulter, USA), and incubated in the dark at room temperature for $20 \mathrm{~min}$. DNA was then stained, and RNA was removed by mixing DNA Prep Stain in the dark at room temperature for another $20 \mathrm{~min}$. The stained cells were then analyzed for DNA content by the BD LSR II (BD Biosciences, USA).The proportion of cells in the S, G1 and G2 phase of the cell cycle was calculated using ModFit LT software (Verity Software House, USA).

\section{Immunoblotting}

Cells were lysed in RIPA lysis buffer $(50 \mathrm{mM}$ Tris- $\mathrm{HCl}$ (pH 7.4), $150 \mathrm{mM} \mathrm{NaCl}, 1 \%$ Triton X-100, 1\% sodium deoxycholate, $0.1 \%$ SDS, $1 \mathrm{mM}$ PMSF). The extracted proteins were separated by SDS-PAGE and transferred to PVDF membranes. After being blocked by TBS-T buffer containing $5 \%$ non-fat powder milk for $2 \mathrm{~h}$, the membranes were immunoblotted using primary antibody against vascular endothelial growth factor (VEGF) (1:1000, Santa Cruz, CA, USA), matrix metalloproteinase-9 (MMP-9) (1:1000, Santa Cruz) and hypoxia-inducible factor-1a (HIF-1a) (1:1000, Santa Cruz) overnight. HRP-conjugated goat anti-rabbit or anti-mouse IgG antibody was used as secondary antibody. $\beta$-actin (1:5000, Santa Cruz) was used as an internal control. Immunoreactive bands on the blots were visualized with enhanced chemiluminescence reagent ECL kit (Biological Industries, Beit Haemek, Israel).

\section{Statistical analysis}

All values in the present study were reported as Mean \pm SD from three independent experiments repeats. Two-sided student's unpaired test was used for statistical analyses. $P$ values less than 0.05 were considered statistically significant. SPSS for Windows version 11.3 (SPSS Inc, Chicago, Ill, United States) was used for analysis.

\section{Results}

\section{Down-regulation of miR-I 26 in hypox- ia-induced RF/6A cells}

The RNA and protein expressions of HIF-1a increased in hypoxic-treated RF/6A cells compared with cells under normoxia (Supplementary Material: Figure S1). To explore the possibility that miR-126 may participate in hypoxia-induced angiogenesis, we compared the expression of miR-126 between control $\mathrm{RF} / 6 \mathrm{~A}$ cells and hypoxic-induced RF/6A cells using real-time quantitative PCR. As shown in Figure 1, miR-126 expression significantly decreased at $6 \mathrm{~h}$ and $24 \mathrm{~h}$ after hypoxia treatment in a time-dependent manner compared with control. The expression of miR-126 in RF/6A cells was diminished by $>100$-fold after $24 \mathrm{~h}$ of hypoxia treatment compared to the normoxic control.

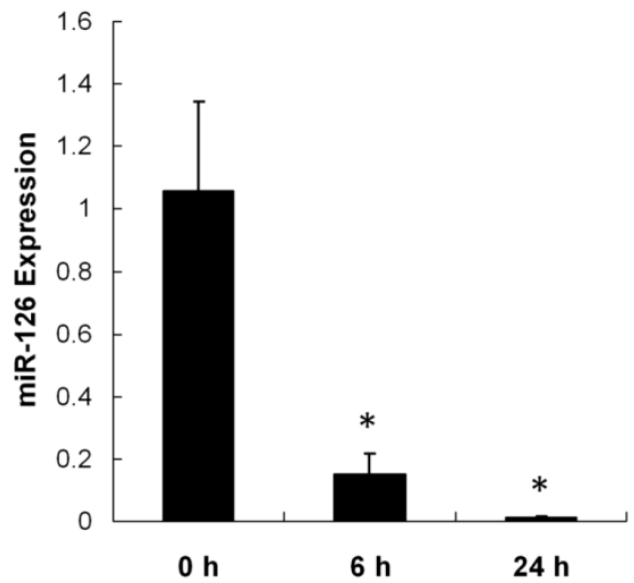

Fig I. Expression of miR-I26 in vitro. One milliliter of cells $\left(I \times 10^{5}\right.$ cells/well) were plated into one well of a six-well culture plate. Hypoxic cultures were transferred for $6 \mathrm{~h}$ and $24 \mathrm{~h}$ in a hypoxic incubator $\left(1 \% \mathrm{O}_{2}\right.$, $5 \% \mathrm{CO}_{2}, 94 \% \mathrm{~N}_{2}$ labeled hypoxia). Parallel cultures were kept in normal oxygen levels. miR- 126 expression significantly decreased at $6 \mathrm{~h}$ and $24 \mathrm{~h}$ after hypoxia treatment in a time-dependent manner compared with control. The expression of miR-126 in RF/6A cells was diminished by > 100 -fold after $24 \mathrm{~h}$ of hypoxia treatment compared with normoxic control. Data were presented as the mean \pm SD of three independent experiments. $* P<0.05$.

\section{Down-regulation of $\mathrm{miR}-\mathrm{I} 26$ in retina tissue of streptozotocin-induced diabetic rats}

We tested miR-126 expression in the retina tissue of STZ-induced diabetic rats 3 month after the initial establishment of the animal model. The photomicrographs (HE x 200) of diabetic rats depicted the blood vessel of the retina (Figure 2). Non-diabetic animal showed a normal vasculature, whereas significant widening of vascular basement membrane was seen in diabetic rats. The reduction of miR-126 levels by 2 -fold was detected in the retina of diabetic rats (Figure 3). These data showed that miR-126 levels were attenuated in hypoxic RF/6A cells and diabetic retina. 

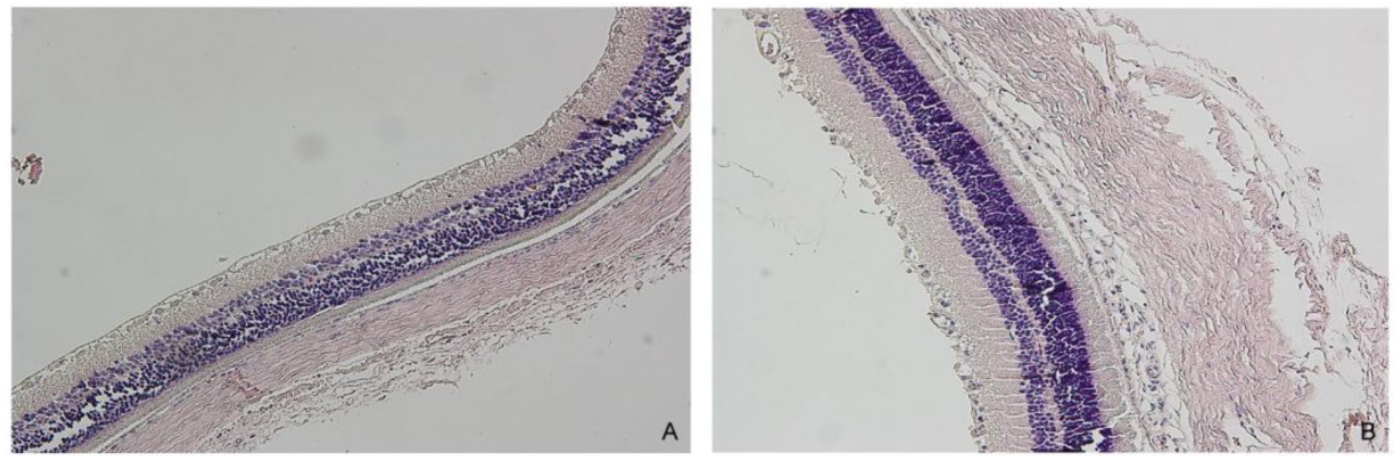

Fig 2. Representative pictures from control and diabetic retina $(H E \times 200)(n=7)$. For the induction of diabetes, the rats were injected with streptozotocin (STZ). The control rats were injected with the citrate buffer. Only rats with blood glucose values $\geq 400 \mathrm{mg} / \mathrm{dl}$ were used as diabetic rats. A. Control retina. B. Diabetic retina showed the widening of the vascular basement membrane. Non-diabetic rat showed a normal vasculature, whereas significant widening of vascular basement membrane was seen in diabetic rat.

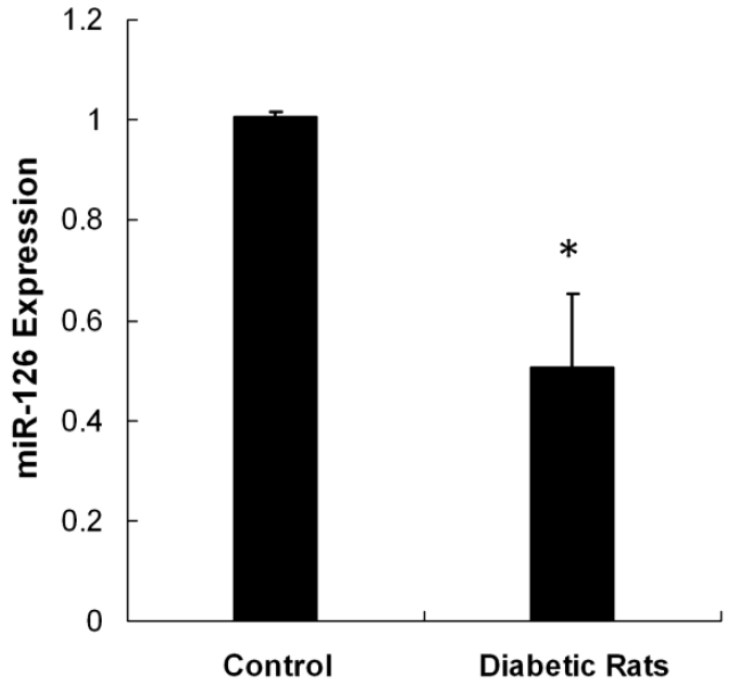

Fig 3. Expression of miR-126 in vivo. Total RNA was extracted from diabetic or control retinas $(n=8)$. Real time PCR was performed and analyzed for miR- 126 expression. The reduction of miR- 126 levels by 2 -fold was detected in the retina of diabetic rats. miR-126 level was attenuated in diabetic retina. Data were presented as the mean \pm SD of three independent experiments. $* P<0.05$.

\section{Normoxia Hypoxia}

\section{VEGF}

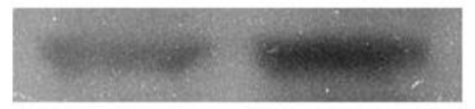

MMP-9

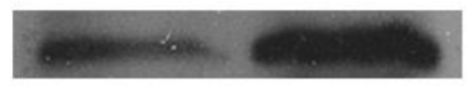

\section{$\beta$-actin}

Fig 4. Expressions of VEGF and MMP-9 in control and hypoxia-induced RF/6A cells. The RF/6A cells were kept in hypoxic incubator or normoxic levels for $24 \mathrm{~h}$. The protein expressions of VEGF and MMP-9 were analyzed by immunoblotting. The protein expression of VEGF and MMP-9 was significantly higher in hypoxia-induced RF/6A cells than that in normoxia after $24 \mathrm{~h}$ treatment. It was one representative blot of three independent experiments.

\section{Increased VEGF and MMP-9 expression levels in hypoxia-induced RF/6A cells}

As VEGF has been suggested to be an important target gene regulated by miR-126 [10], we examined the protein expression of VEGF in control and hypoxia-induced RF/6A cells by immunoblotting. At 24 $\mathrm{h}$ after treatment, the hypoxic cells showed remarkably higher expression of VEGF than normoxic cells (Figure 4). We also detected the protein expression of MMP-9 in RF/6A cells after $24 \mathrm{~h}$ of hypoxia. As shown in Figure 4, hypoxia led to a significant increase in the MMP-9 protein level.

\section{Regulation of cell cycle by miR-I 26 in hypox- ia-induced RF/6A cells}

To evaluate the functional role of miR-126 in hypoxia-induced RF/6A cells, we transfected RF/6A cells with miR-126-mimics, miR-126-inhibitors, or their negative controls, and used flow cytometry to examine the DNA profiles of asynchronous populations in hypoxia-treated cells. As shown in Figure 5, $24 \mathrm{~h}$ of hypoxia significantly reduced the percentage of RF/6A cells in S phases in miR-126-mimic group compared to the negative control group. Inhibition of miR-126 slightly increased the percentage of RF/6A cells in S phases, but it didn't reach statistical significance. These data implied the negative role of miR-126 in $S$ phase under hypoxic condition.

\section{Changes in the VEGF and MMP-9 expression levels in hypoxia-induced RF/6A cells pre- treated with miR-I 26-mimics or miR-I 26-inhibitors}

We examined the effect of miR-126 on VEGF and MMP-9 expression in hypoxia-induced RF/6A cells pretreated with miR-126-mimics, miR-126-inhibitors, or their negative controls by immunoblotting. Pretreatment with miR-126-mimics decreased VEGF and MMP-9 expression, whereas pretreatment with 
miR-126-inhibitors increased VEGF and MMP-9 expression in hypoxia-induced RF/6A cells (Figure 6). However, the miR-126-induced down-regulation on VEGF and MMP-9 was very moderate, and the dif- ference was not significant under normoxic conditions (Supplementary Material: Figure S2). These results suggested that miR-126 may act as a negative regulator of VEGF and MMP-9 under hypoxia.
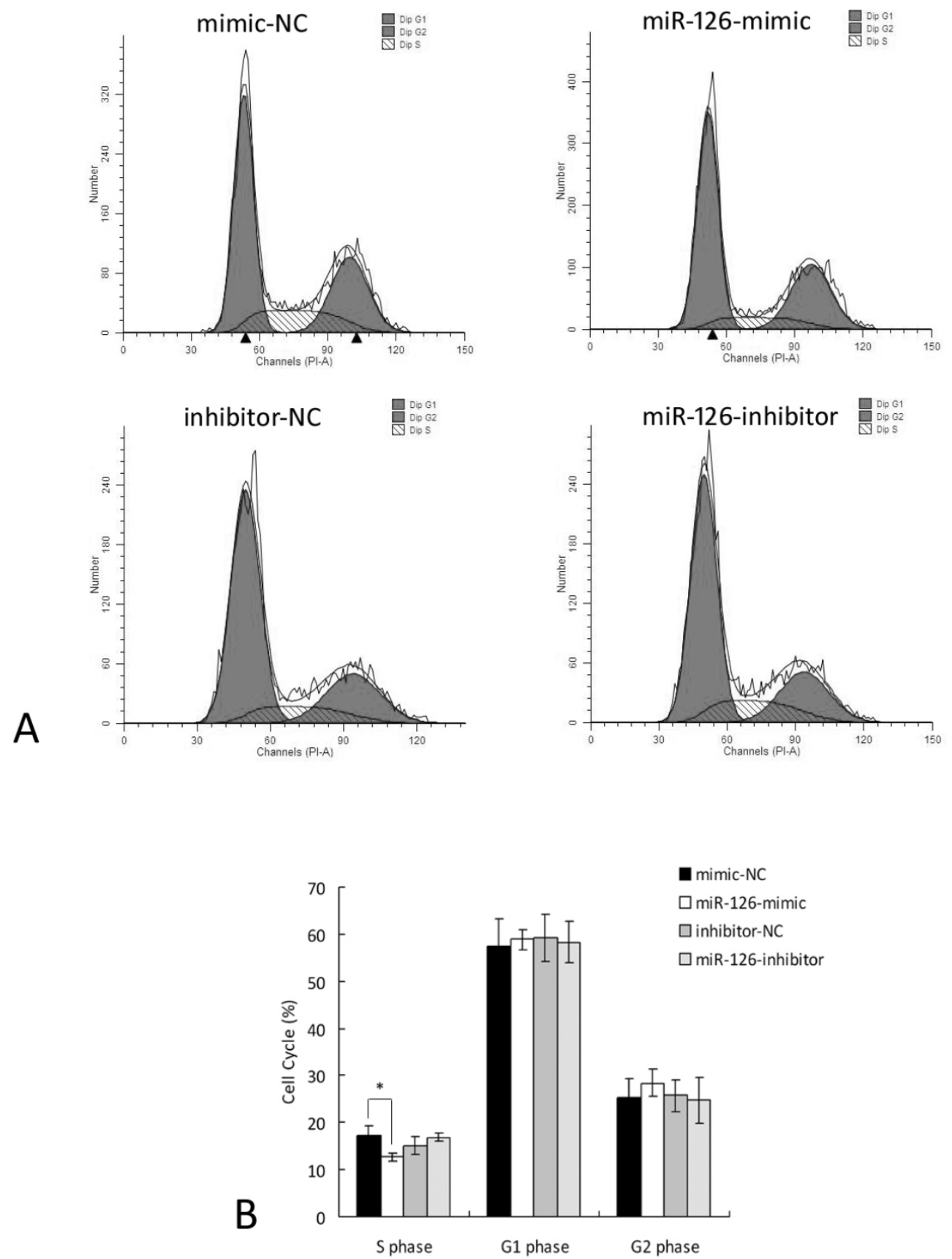

Fig 5. Over- and down-expression of miR-126 alters the proportion of cells in $S$ phases under hypoxia. The RF/6A cells were treated with miR-126-mimics, miR-126-inhibitors, or negative controls for $6 \mathrm{~h}$ and then kept in hypoxic incubator or normoxic levels for $24 \mathrm{~h}$. The cells were stained and analyzed for cell cycle. A. The representative FACS plots displayed differences in cell cycle phases of hypoxia-induced RF/6A cells pretreated with miR-I26-mimics or miR-126-inhibitors. B. Cell cycle analysis demonstrated that $24 \mathrm{~h}$ of hypoxia significantly reduced the percentage of cells in S phases in miR-126-mimic group compared with negative control group. Data were presented as the mean \pm SD of three independent experiments. $* P<0.05$. 


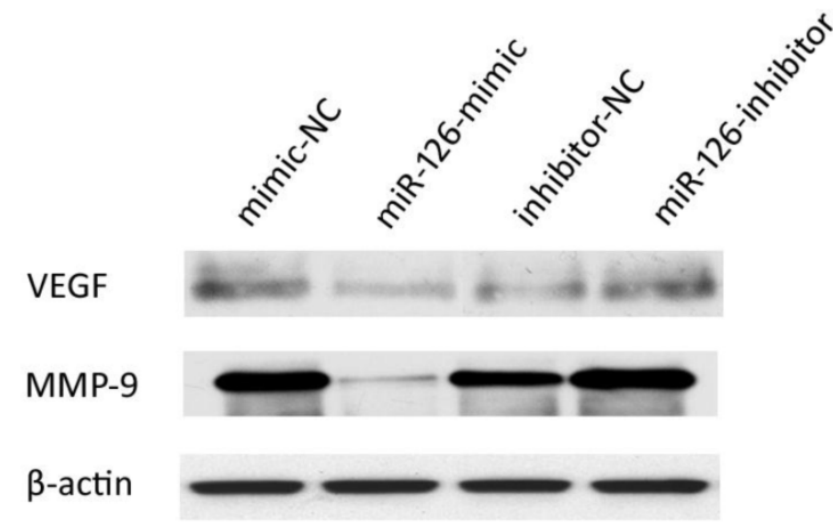

Fig 6. Effect of miR-126 on the VEGF and MMP-9 expressions in hypoxia-induced RF/6A cells pretreated with miR-126-mimics or miR-I26-inhibitors. The RF/6A cells were treated with miR-126-mimics, miR-I26-inhibitors, or negative controls for $6 \mathrm{~h}$ and then kept in hypoxic incubator levels for $24 \mathrm{~h}$. The protein expressions of VEGF and MMP-9 were analyzed by immunoblotting. Pretreatment with miR-I26-mimics decreased VEGF and MMP-9 expression, whereas pretreatment with miR-126-inhibitors increased VEGF and MMP-9 expression in hypoxia-induced RF/6A cells. It was one representative blot of three independent experiments.

\section{Discussion}

Hypoxia-induced retinal neovascularization is a key pathological alteration in diabetic retinopathy and other serious retinal diseases [12]. Diabetes causes hypoxia and ischemia of the retina and triggers a series of structural and functional alterations in the retinal endothelial cells. VEGF has been suggested as one of the most potent angiogenic factors in retinal neovascularization $[13,14]$. Others have shown that VEGF is produced by several cell types within the eye; sources could be retinal pigment endothelial cells, glial cells, retinal capillary pericytes, endothelial cells, Müller cells, and ganglion cells [15].

miR-126, an endothelial-specific miRNA, has recently been discovered to play a major role in vascular development and angiogenesis [16, 17]. The effect of miR-126 in angiogenesis remains controversial. Previous reports focused on the role of miR-126 in normal ECs $[9,16,18]$. Deletion of miR-126 causes loss of vascular integrity and produces defects in endothelial cell proliferation, migration, and angiogenesis [9]. However, recent investigations have revealed the beneficial effect of miR-126 in cases of pathological vascularization [19]. miR-126 was found as a tumor suppressor gene in lung cancer cells with an inhibitory effect on VEGF expression by targeting a binding site in its mRNA 3'UTR [10]. It has been recently demonstrated that miR-126 was down-regulated in the blood of patients with coronary artery disease [20] and also in endothelial progenitor cells from diabetic patients [11]. The deregulation of miR-126 impaired endothelial progenitor cell-mediated functions [11]. In this study, RF/6A cells cultured under hypoxic conditions had decreased miR-126 levels compared to cells maintained under normoxic conditions. The decreased miR-126 expression was hypoxic time-dependent. Moreover, reduced miR-126 expression was also detected in diabetic retina. These data are consistent with those reported by Zampetaki et al., who reported decreased plasma miR-126 expression in patients with type 2 diabetes mellitus [21].

In the functional analysis, we demonstrated that up-regulated miR-126 expression inhibited the percentage of RF/6A cells in S phase and caused the arrest of the cell cycle, which indicates an angiogenesis suppressor role for miR-126 under hypoxia conditions. However, miR-126 inhibitors did not cause significant changes in the cell cycle. One possible explanation might be that miR-126 inhibitors do not have as potent of an effect as miR-126 mimics because of the extremely low expression of miR-126 under hypoxic condition.

MMPs are regarded as the main critical proteins that assist in the migration of endothelial cells during angiogenesis. The initial steps contributing to degradation of the extracellular matrix surrounding the endothelial cells is induced by MMPs [3, 22]. MMP-9, a member of the MMP family, also known as gelatinases, can degrade important substrates such as collagen IV, laminin and fibronectin, which are major components of vascular lamina [23]. In this study, hypoxia induced a significant, sustained increase in the protein levels of VEGF and MMP-9, which are closely associated with neovascularization. To further gauge the regulative effect of miR-126, we detected the protein expression of VEGF and MMP-9 after incubation with miR-126 mimics or inhibitors in RF/6A cells under hypoxic conditions. miR-126 mimics significantly inhibited the expression of VEGF and MMP-9 in hypoxic RF/6A cells while miR-126 inhibition slightly increased their expression. The miR-126-induced down-regulation on VEGF and MMP-9 was very moderate, and the difference was not significant under normoxic conditions. Taken together, miR-126 may be considered as a potential therapeutic target for hypoxic neovascularization.

In conclusion, the present study showed a reduction of miR-126 expression under hypoxic conditions such as one would find in diabetic retinas. Moreover, we found that miR-126 may halt the processes of angiogenesis via arrest of the cell cycle and inhibition of VEGF and MMP-9 expression. The findings of this study highlight an important clinical implication of miR-126 in hypoxia-associated retinal neovascularization. 


\section{Supplementary Material}

Fig.S1-Fig.S2.

http://www.medsci.org/v11p0017s1.pdf

\section{Acknowledgments}

This study was supported by Zhejiang Key Innovation Team Project (No.2009R50039), Doctoral Fund of Ministry of Education of China (No.20100101120135), Key Lab Fund of Zhejiang Province, China (No.2011E10006), Key Program of National Natural Science Foundation of China (No.81130018) and Project of National Clinical Key Discipline of Chinese Ministry of Health.

\section{Competing Interests}

The authors have declared that no competing interest exists.

\section{References}

1. Nyengaard JR, Ido Y, Kilo C, Williamson JR. Interactions between hyperglycemia and hypoxia: implications for diabetic retinopathy. Diabetes. 2004; 53: 2931-8. doi:53/11/2931 [pii].

2. Grammas P, Riden M. Retinal endothelial cells are more susceptible to oxidative stress and increased permeability than brain-derived endothelial cells. Microvasc Res. 2003; 65: 18-23. doi:S002628620200016X [pii].

3. Folkman J, Shing Y. Angiogenesis. J Biol Chem. 1992; 267: 10931-4.

4. Huang Y, Shen XJ, Zou Q, Wang SP, Tang SM, Zhang GZ. Biological functions of microRNAs: a review. J Physiol Biochem. 2011; 67: 129-39. doi:10.1007/s13105-010-0050-6.

5. Kozomara A, Griffiths-Jones S. miRBase: integrating microRNA annotation and deep-sequencing data. Nucleic Acids Res. 2011; 39: D152-7. doi:gkq1027 [pii]10.1093/nar/gkq1027.

6. Caporali A, Emanueli C. MicroRNA regulation in angiogenesis. Vascul Pharmacol. 2011; 55: 79-86. doi:S1537-1891(11)00070-X [pii]10.1016/j.vph.2011.06.006.

7. Kuehbacher A, Urbich C, Dimmeler S. Targeting microRNA expression to regulate angiogenesis. Trends Pharmacol Sci. 2008; 29: 12-5. doi:S0165-6147(07)00277-5 [pii]10.1016/j.tips.2007.10.014.

8. Fitch MJ, Campagnolo L, Kuhnert F, Stuhlmann H. Egfl7, a novel epidermal growth factor-domain gene expressed in endothelial cells. Dev Dyn. 2004; 230: 316-24. doi:10.1002/dvdy.20063.

9. Wang S, Aurora AB, Johnson BA, Qi X, McAnally J, Hill JA, et al. The endothelial-specific microRNA miR-126 governs vascular integrity and angiogenesis. Dev Cell. 2008; 15: 261-71. doi:S1534-5807(08)00281-5 [pii]10.1016/j.devcel.2008.07.002.

10. Liu $B$, Peng $X C$, Zheng $X L$, Wang $J$, Qin YW. MiR-126 restoration down-regulate VEGF and inhibit the growth of lung cancer cell lines in vitro and in vivo. Lung Cancer. 2009; 66: 169-75. doi:S0169-5002(09)00012-9 [pii]10.1016/j.lungcan.2009.01.010.

11. Meng S, Cao JT, Zhang B, Zhou Q, Shen CX, Wang CQ. Downregulation of microRNA-126 in endothelial progenitor cells from diabetes patients, impairs their functional properties, via target gene Spred-1. J Mol Cell Cardiol. 2012; 53: 64-72. doi:10.1016/j.yjmcc.2012.04.003 S0022-2828(12)00150-2 [pii].

12. Frank RN. Diabetic retinopathy. N Engl J Med. 2004; 350: 48-58. doi:10.1056/NEJMra021678 350/1/48 [pii].

13. Aiello LP, Northrup JM, Keyt BA, Takagi H, Iwamoto MA. Hypoxic regulation of vascular endothelial growth factor in retinal cells. Arch Ophthalmol. 1995; 113: $1538-44$.

14. Adamis AP, Shima DT, Tolentino MJ, Gragoudas ES, Ferrara N, Folkman J, et al. Inhibition of vascular endothelial growth factor prevents retinal ischemia-associated iris neovascularization in a nonhuman primate. Arch Ophthalmol. 1996; 114: 66-71.

15. Duh E, Aiello LP. Vascular endothelial growth factor and diabetes: the agonist versus antagonist paradox. Diabetes. 1999; 48: 1899-906.

16. Fish JE, Santoro MM, Morton SU, Yu S, Yeh RF, Wythe JD, et al. miR-126 regulates angiogenic signaling and vascular integrity. Dev Cell. 2008; 15: 272-84. doi:S1534-5807(08)00287-6 [pii]10.1016/j.devcel.2008.07.008.

17. Hartmann D, Thum T. MicroRNAs and vascular (dys)function. Vascul Pharmacol. 2011; 55: 92-105. doi:S1537-1891(11)00072-3 [pii]10.1016/j.vph.2011.07.005.

18. Kuhnert F, Mancuso MR, Hampton J, Stankunas K, Asano T, Chen CZ, et al. Attribution of vascular phenotypes of the murine Egfl7 locus to the microRNA miR-126. Development. 2008; 135: 3989-93. doi:dev.029736 [pii]10.1242/dev.029736.
19. Bai Y, Bai X, Wang Z, Zhang X, Ruan C, Miao J. MicroRNA-126 inhibits ischemia-induced retinal neovascularization via regulating angiogenic growth factors. Exp Mol Pathol. 2011; 91: 471-7. doi:S0014-4800(11)00056-6 [pii]10.1016/j.yexmp.2011.04.016.

20. Fichtlscherer S, De Rosa S, Fox H, Schwietz T, Fischer A, Liebetrau C, et al. Circulating microRNAs in patients with coronary artery disease. Circ Res. 2010; 107: 677-84. doi:CIRCRESAHA.109.215566 [pii]10.1161/CIRCRESAHA.109.215566.

21. Zampetaki A, Kiechl S, Drozdov I, Willeit P, Mayr U, Prokopi M, et al. Plasma microRNA profiling reveals loss of endothelial miR-126 and other microRNAs in type 2 diabetes. Circ Res. 2010; 107: 810-7. doi:CIRCRESAHA.110.226357 [pii]10.1161/CIRCRESAHA.110.226357.

22. Xu J, Zhu D, Sonoda S, He S, Spee C, Ryan SJ, et al. Over-expression of BMP4 inhibits experimental choroidal neovascularization by modulating VEGF and MMP-9. Angiogenesis. 2012; 15: 213-27. doi:10.1007/s10456-012-9254-4.

23. Nguyen M, Arkell J, Jackson CJ. Human endothelial gelatinases and angiogenesis. Int J Biochem Cell Biol. 2001; 33: 960-70. doi:S1357-2725(01)00007-3 [pii]. 